OS11 (1) - 1 (OS11W0187)

\title{
Effects of Frictional Wear Characteristics and Microstructure on Fretting Fatigue Strength of High Workable Titanium Alloy, Ti-4.5AI-3V-2Mo-2Fe
}

\author{
Junji Takeda, Graduate school of Production Systems Engineering, Toyohashi Univ. of Tech., Toyohashi, Japan \\ Mitsuo Niinomi, Dept. of Production Systems Engineering, Toyohashi Univ. of Tech., Toyohashi, Japan \\ Toshikazu Akahori, Dept. of Production Systems Engineering, Toyohashi Univ. of Tech., Toyohashi, Japan
}

Recently, Ti-4.5Al-3V-2Mo-2Fe, which is an alpha + beta type titanium alloy with excellent super plastic forming characteristics, has been developed for aircraft applications. Fretting fatigue fracture, which is caused by the combination of cyclic fatigue stress and frictional wear, is highly expected for aircraft materials. Microstructure of Ti-4.5Fe-3V-2Mo- $2 \mathrm{Fe}$ is variously changed by heat treatments. Therefore, the effects of frictional wear characteristics and microstructural factors such as area fraction and diameter of primary alpha phase on fretting fatigue strength of Ti-4.5Al-3V-2Mo- $2 \mathrm{Fe}$ conducted with various annealing treatments were investigated in this study.

Fretting fatigue life of Ti-4.5Al-3V-2Mo-2Fe is hardly affected by the area fraction and diameter of primary alpha phase in the low cycle fretting fatigue life region. On the other hand, the fretting fatigue limit of Ti-4.5Al-3V-2Mo-2Fe tends to increase with decreasing the area fraction of primary alpha phase up to $29.7 \%$ and average diameter of primary alpha phase up to 2.32 micrometer, and to be constant below the area fraction of primary alpha phase of $29.7 \%$ and average primary alpha phase diameter of 2.32 micrometer. Fretting fatigue crack of Ti-4.5Al-3V-2Mo-2Fe initiates at a boundary between stick region and slip region where the maximum frictional force generates at the earlier stage of fretting fatigue. After the fretting fatigue crack grows to be a few hundreds micrometer in depth and width, the growth behavior of fretting fatigue crack would be similar to that of plain fatigue crack.

The weight loss of Ti-4.5Al-3V-2Mo-2Fe conducted with various annealing treatments tends to increase with decreasing the hardness. The estimated weight loss of slip regions of fretting fatigue specimens of Ti-4.5Al-3V-2Mo-2Fe conducted with various annealing treatments obtained at high cycle fatigue life region tends to increase with increasing the length of the slip region. The fretting fatigue life of Ti-4.5Al-3V-2Mo-2 $\mathrm{Fe}$ conducted with various annealing treatments tends to decrease with increasing the length of the slip region and increasing the estimated weight loss of the slip region. The depth of the crack growth zone caused by the contact pressure at high cycle fatigue life region tends to increase with increasing the estimated weight loss of the slip region. The fretting fatigue life of Ti-4.5 Al- $3 \mathrm{~V}-2 \mathrm{Mo}-2 \mathrm{Fe}$ conducted with various annealing treatments tends to decrease with increasing the estimated weight loss of the slip region and increasing the depth of the crack growth zone caused by the contact pressure. 